\title{
THE NEW RIEMANN-WEBER
}

Die Differential- und Integralgleichungen der Mechanik und Physik, the 7th Edition of Riemann-Weber's Partiellen Differentialgleichungen der mathematischen Physik, edited by P. Frank and R. von Mises. Erster, mathematischer Teil, edited by $\mathrm{R}$. von Mises with the assistance of L. Bieberbach, C. Carathéodory, R. Courant, K. Loewner, H. Rademacher, E. Rothe, C. Szegö. Braunschweig, Vieweg, 1925. 687 pp. +76 figures. Paper, 40 Rentenmark; bound, 44 Rentenmark.

As a compliment to the memory of Riemann and $\mathrm{H}$. Weber, the present work is nominally issued as the seventh edition of Riemann-Weber, The Partial Differential Equations of Mathematical Physics. The fourth edition of Riemann's treatise under the above title was Heinrich Weber's first contribution. The fifth edition (Weber's second) was issued in 1910 and the sixth edition was a mere reprint of the fifth. H. Weber before his death had planned an edition which should be entirely rewritten with the cooperation of a group of younger mathematicians and physicists. His chief collaborators were to be his son, R. Weber, and J. Wellstein. The latter two did not survive the elder Weber very long, and the work was then undertaken by $\mathrm{R}$. von Mises with the cooperation of the seven listed above.

In the main the present plan differs from the original in a division into a mathematical part (the present volume under review), and a second, a physical part announced for an early appearance. The second part is to be edited by P. Frank and written with the help of R. Furth, A. Sommerfeld, F. Noether, Th. v. Karman, and E. Trefftz. Though written by eight collaborators, the present work exhibits a degree of unity far beyond what might be expected. To be sure, the field as a whole lends itself by its very nature to systematic treatment and unification. But the skill with which this is accomplished is due chiefly to the labor of the editor and chief contributor, von Mises.

The following is a summary of the table of contents by chapters.

Part I. Fundamental preliminary theory. 1. Real functions. 2. Linear forms. 3. Complex variables. 4. Infinite series and products. 5. Calculus of variations.

Part II. Ordinary differential equations. 1. Problems involving initial conditions. 2. Boundary value problems of the second order. 3. Particular functions corresponding to second order boundary value problems. 4. Development of arbitrary functions. 5. Developments in Bessel's functions.

Part III. Integral equations and potential. 1. Summary of problems and results. 2. Solution of integral equations. 3. Applications of integral equations to boundary value problems. 4. Potential.

Part IV. Partial differential equations. 1. Problems involving initial values. 2. Potential in the plane. 3. Potential in three-space. 4. Boundary value problems for partial differential equations of the second order. 
5. Other problems in partial differential equations. 6. Calculus of variations and boundary value problems.

There is some overlapping in the various chapters, but this is of a helpful kind. Beginning with the fundamentals of the theory of functions of a real variable and of a complex variable, through the calculus of variations and vector and tensor analysis, the work proceeds with a definite end in view. All of these matters are to join on to specific topics in mathematical physics.

It is clear to the reviewer that the present plan purposes not merely to rearrange material but to offer a well organized presentation of the mathematical "tool-box" of modern theoretical physics. That the reader is carried well beyond undergraduate courses in calculus and differential equations is attested by such topics as "functions of limited variation," "Stieltjes and Lebesgue integrals," "conformal mapping," "extremals," "canonical coordinates," "contact transformations," "characteristic functions," "Dirichlet's principle," "infinitely many variables," etc. The volume is indeed of value alike to the worker in physics and to the mathematical specialist, even though not all the recent advances in the various fields, especially results of a very particular nature, are included. But in the main, where the method is of importance, a detailed discussion is at hand. For example, for special results, the present volume is not as complete as a book which appeared a year earlier (1924) written by one of the present writers, R. Courant, Methoden der Mathematischen Physik, which forms volume XII of Die Grundlehren der mathematischen Wissenschaften in Einzeldarstellungen. The present book, however, offers a wider perspective. The treatment is not encyclopedic, nor exhaustive, but it lays a broad foundation and provides a sufficient framework around which courses in "pure" or "physical" mathematics may be built.

To give a detailed account of the contents of the book would be to rehearse not only the recent developments of the past thirty years work which may be included under the heading of differential and integral equations, but most of the modern and classical developments of analysis. The names of von Mises, Bieberbach, Carathéodory and Courant, well known both as research workers and excellent expositors, as well as the others, are the best recommendation one can give for the value of the present work. Supplementary references to more extensive and special treatises are to be found at the end of most of the chapters, together with occasional mention of original memoirs.

H. J. EtTlinger 